\title{
La Voce dei Pazienti Medici di base, aggiornatevi sul rene policistico!
}

Sulla pagina Facebook di AIRP Onlus, un coro di esperienze negative con i medici di base: impreparati o superficiali nell'affrontare una patologia che spesso conoscono poco e male. Per loro, i corsi di aggiornamento organizzati da AIRP dovrebbero essere obbligatori...

M.S.: leri una mia conoscente mi chiede del trapianto di mio figlio, al quale io ho fatto da donatrice. lo le spiego, lei ascolta e poi mi racconta, a sorpresa, che anche lei ha i reni policistici e anche molte cisti al fegato e anche sua sorella gemella... La signora fa i controlli solo dal suo medico di base, il quale genericamente le dice che sia lei che la sorella finiranno in tarda età a dover fare la dialisi. E basta! Nessun altro consiglio, neanche il più semplice, come invitarla a rivolgersi a un nefrologo. Ha anche un bambino di 11 anni... Rimango sempre spiazzata dall'impreparazione dei medici di famiglia. Ho dato a quella signora i nostri depliant e il numero di Luisa e l'ho invitata a visitare il sito AIRP per leggere e informarsi. Dopo più di trent'anni, ancora e sempre le stesse storie... Ma non ci si può meravigliare! II mio ex medico di base, quando I'ho invitato a partecipare a un nostro Convegno, informandolo anche dei crediti ECM, mi ha risposto: "Ah no, già perdo un sacco di tempo per i corsi di aggiornamento... Di sabato proprio no". Peccato che si stesse rivolgendo a una madre, il cui figlio era in attesa di trapianto. Così, ha perso in un attimo sei pazienti e, cosa ancora peggiore, è rimasto nella sua comprovata ignoranza...

P.G.: Come hai ragione! II mio medico di base, quando vado per qualsiasi cosa, non fa mai, dico mai, riferimento alla mia patologia. Mai un consiglio, mai un'opinione. II nulla più assoluto!

M.S.: lo penso che sia comprensibile che il medico di base non abbia la stessa conoscenza e gli strumenti di uno specialista, ma non ha neanche il buongusto di documentarsi quando un suo paziente è affetto dalla patologia! Questa mi sembra una grave mancanza di professionalità.

D.B.: Con il mio medico di base, a volte faccio fatica a capire... Devo fare sempre dei controlli richiesti dal nefrologo e lui non riesce neppure a prescrivermi gli esami giusti... Oggi ho dovuto pagare la maggior parte degli esami, visto che ha sbagliato sia l'esenzione che la prescrizione... Sarebbe da denuncia...

M.S.: Posso darti un consiglio? Cambia medico! to ho aspettato tanto, con tutte le problematiche che racconti tu... Inutile, ci sono già i problemi legati alla malattia, perché combattere anche per altro?
S.G.: La prevenzione dovrebbe partire dai medici di famiglia. I corsi di aggiornamento non dovrebbero essere con crediti, ma alla rovescia: non ti aggiorni? Allora ti togliamo mille euro di stipendio...

S.V.: Non parliamo di medici di famiglia, il mio è uno scandalo! Quando hai dei pazienti con malattie poco conosciute, i corsi di aggiornamento dovrebbero essere obbligatori! Invece se ne fregano e pensano di saperne di più dei nefrologi...

M.G.: II mio medico, più di 20 anni fa, mi ha fatto fare sempre gli esami delle urine e del sangue con la creatinina, ma anche lui mi rassicurava che avrei avuto i problemi più gravi solo da vecchia. E poi, dopo pochi anni, era molto meravigliato quando la mia malattia galoppava...

L.P.: Questa "prassi" è ancora diffusissima tra i medici di base e non solo, purtroppo siamo costretti ad arrangiarci, con visite private, impegnandoci in ricerche e cercando più informazioni possibili. Per fortuna che c'è l'AIRP a darci sostengo e conoscenza!

A.l.: lo ho 39 anni e, finora, ho sempre incontrato medici ottimisti, per non dire poco informati sulla nostra patologia e non faccio distinzione tra generici e specialisti. L'ultimo lunedì scorso: esimio Professore in Allergologia Pediatrica dove ho portato mio figlio (policistico). Mi ha illuminato, sostenuto e incoraggiato per quanto riguarda l'intolleranza al latte di Andrea, ma, quando ha visto la sua ecografia, mi sono cadute le braccia. Queste le sue parole: "Vabbè ha i reni policistici, e questo non ce ne frega, se li tiene, tanto non gli succede niente"!!! Ho provato a ribattere, ma lui: "Signora, non badi alle chiacchiere dei nefrologi, per loro siete solo dei casi da studiare... Non è necessario fare controlli, mio padre è morto ultranovantenne e solo qualche anno prima abbiamo scoperto che era policistico". Amici, credetemi, avrei voluto ribattere, ma mi ha fatto quasi pena...

A.M.: A Natale regalagli il libro A.I.R.P.!!!

A.I.: Il medico "ottimista" per me è colui che, conoscendo bene la malattia, te la illustra nei suoi mille risvolti e ottimisticamente ti espone anche la possibilità di arrivare alla vecchiaia discretamente... Quando, invece, parlo di "incompetenti", mi riferisco a coloro i quali, senza conoscere la patologia, ti dicono che le cisti non creano alcun problema! 
T.S.: lo mi ricordo quando ho scoperto di avere questa patologia. Ero da un urologo e scoppiai a piangere pensando a mio padre... II medico e le assistenti mi guardarono come se venissi da un altro pianeta!!!

D.B.: II mio nefrologo scrive sempre che ho i reni policistici e ho un esame istologico che conferma la diagnosi, ma il medico di base, sulle richieste, scrive sempre rene multicistico e, ogni volta che lo faccio notare, mi risponde che non fa differenza...

A.R.: Come fanno a dire che i reni policistici non creano problemi? A me si era talmente ingrandito che me lo hanno dovuto togliere e solo per altri problemi non mi hanno tolto anche l'altro rene.
C.B.: Fatevi sempre consigliare dagli specialisti nefrologi e non affidatevi completamente ai medici di base e/o di famiglia che pigliano delle cantonate. Lo so per esperienza. A me non mi visita nemmeno il medico di famiglia e sì che dovrebbe! Anzi: spargete sempre la voce, fra tutti i vostri amici e amiche, che è importante fare prevenzione e controllare sempre i reni. $\mathrm{E}$, infine, informatevi il più possibile, e non solo sul web, ma andando di persona dal nefrologo. Ciao, un abbraccio a tutti!

Published online: March 3, 2016 